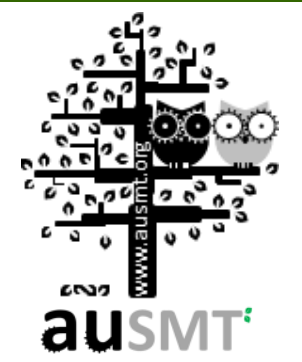

\title{
Design of Wireless Sensors for Intelligent Manufacture Monitoring
}

\author{
Chia-Chan Chang1, 3, *, Chung-Yi Liu', Kui-Hua Huang², \\ and Guo-Hua Feng2, 3 \\ ${ }^{1}$ Department of Electrical Engineering, National Chung-Cheng University, Taiwan \\ ${ }^{2}$ Department of Mechanical Engineering, National Chung-Cheng University, Taiwan \\ ${ }^{3}$ Advanced Institute of Manufacturing with High-tech Innovations, National Chung-Cheng University, Taiwan \\ (Received 12 February 2014; Accepted 26 March 2014; Published on line 1 September 2014) \\ *Email: 1 \\ DOI: 10.5875 /ausmt.v4i3.604
}

\begin{abstract}
Continuous monitoring of manufacturing machinery can allow industrial firms to replace worn parts before they fail, thus preventing production line downtime, but such monitoring requires the development of precise and cost-effective sensing system. This paper demonstrates a wireless sensing system composed of a temperature sensor module and a Wi-Fi transceiver module to monitor the status of ball screws used in industrial machinery. The sensing unit is constructed from a very small $\left(\sim 1 \mathrm{~cm}^{3}\right)$ SMD-type glass PT-1000, while the Wi-Fi module is integrated into with a microcontroller on a printed circuit board smaller than $5 \mathrm{~cm}^{2}$. An experimental setup is implemented on a single-axis ball screw stage using three temperature sensors embedded at different positions. Temperature data are successfully measured and transmitted to a central computer, verifying the feasibility of this proposed system.
\end{abstract}

Keywords: Ball screw; wireless sensor; Wi-Fi

\section{Introduction}

Factory production is increasingly intelligent and automated, using feed-driven machine tool to process work pieces and position cutting tools for unprecedented efficiency and accuracy $[1,2]$. The feed drives in most modern machines are actuated by linear motors or by rotary motors with a ball screw and nut assembly. Feed drives with ball screws are frequently used to carry medium/high loads [3]. The drive configuration is composed of a working table travelling along two parallel linear guides. The working table is positioned by the motor-driven ball screw which is supported by two bearings. The reliable and accurate operation of ball screws is extremely crucial for many applications, such as in mechatronic equipment for the production of semiconductors and printed circuit boards, in robotics, or

www.ausmt.org

Copyright (C) 2014 International Journal of Automation and Smart Technology other precision motion control facilities.

Nevertheless, heavy usage or improper installation of the feed drive will gradually result in wear or even breakage, particularly of moving parts or the feed drive transmission interface [4]. Sudden failure could incur considerable downtime. Currently, wear of such components is usually diagnosed based on the production of unusual sounds or vibrations, or of changes to product quality, but this is dependent on operator experience. A means of objectively, continuously and reliably estimating remaining device life spans would considerably assist manufacturers to arrange maintenance or replacement in advance, thus avoiding breakdowns and downtime.

Since the balls serve as rotating medium within a feed drive, the motion and behavior of these balls provide very useful information, such as degree of wear, lubrication and breakage. Quantifying these parameters 
Table 1. Comparison of Bluetooth, UWB, ZigBee, and Wi-Fi protocols.

\begin{tabular}{|c|c|c|c|c|}
\hline Technology (IEEE standard) & ZigBee 802.15 .4 & Bluetooth 802.15.1 & $W i-F i$ 802.11a/b/g/n & UWB 802.15.3 \\
\hline RF frequency & $868 / 915 \mathrm{MHz}, 2.4 \mathrm{GHz}$ & $2.4 \mathrm{GHz}$ & $\begin{array}{c}5.8 \mathrm{GHz}-\mathrm{a} \\
2.4 \mathrm{GHz}-\mathrm{b} / \mathrm{g} / \mathrm{n},\end{array}$ & 3.1 to $10.6 \mathrm{GHz}$ \\
\hline Data rate $(\mathrm{Mb} / \mathrm{s})$ & 0.25 & $1 \sim 3$ & $11 \sim 105$ & $110 \sim 1600$ \\
\hline Range (m) & $10 \sim 100$ & 10 & $10 \sim 100$ & $4 \sim 20$ \\
\hline Useful BW (MHz) & 0.6 & $1 \sim 3$ & $20-40$ & $120 \sim 1000$ \\
\hline Power consumption & Very low & Low & High & Low \\
\hline Advantages & Power, Cost & Cost, Convenience & Speed, Flexibility & Data capacity \\
\hline
\end{tabular}

and providing appropriate feedback in manufacturing and industrial contexts requires a precise sensing system [5]. Unfortunately, the health status of the feed drive is difficult to diagnose. The balls are particular difficult to monitor given their location within the key components of the feed drive.

Data collection/transmission for further analysis is also an important issue in sensing systems. Traditional wired sensor systems are very costly to deploy and maintain, and wireless technologies such as infrared $[6,7]$ and radio waves [8-15] can allow operators to remotely gather device status information. Among these solutions, the radio frequency (RF)-based technique is particularly well-suited because it can be adaptated to existing accessible wireless devices.

While RF-based wireless devices are widely used in many domains, such systems face challenges in industrial contexts because of electro-magnetic interference due to multiple reflections from metal in the environment. Such interference could seriously degrade data integrity, and deployment of such systems requires repeated on-site testing to determine optimal communications channels. Security is another concern over open, shared wireless channels. To overcome this issue, data can be encrypted to increase the security level, but doing so increases processing complexity and power consumption.

Chia-Chan Chang received her Ph.D. in electrical and computer engineering from the University of California at Davis, in 2003. In Feb. 2004, she joined the faculty of the Dept. of Electrical Engineering at National Chung-Cheng University, Chiayi, Taiwan, where she is currently an Associate Professor. Her research interests include phased arrays and beamforming technologies, mm-wave IC designs, CMOS-MEMS reconfigurable circuits and the radar system applications.

Chun-Yi Liu received his M.S. in electrical engineering from National Chung Cheng University, Chiayi, Taiwan, in 2013. His research interest is the design of wireless data transmission modules.

Guo-Hua Feng received his Ph.D. in electrical engineering from the University of Southern California in 2004. In 2005 he joined the Department of Mechanical Engineering at National Chung Cheng University, Taiwan, and now serves as professor there. His research interests include MEMS industrial applications, machine health monitoring, sensing technologies, and microfabrication processing technologies.

Kui-Hua Huang is an M.S. candidate in the Department of Mechanical Engineering at National Chung Cheng University, Taiwan.
The choice of an adequate wireless technology is crucial. Rapid developments in wireless communication technologies have provided a range of options including RFID, Bluetooth, ZigBee, etc., each with its individual strengths and weaknesses. Wireless sensor networks are categorized as short-range communication networks, which currently includes four widely-used protocols: ZigBee [8, 9], Bluetooth [10, 11], Wireless Fidelity (Wi-Fi) $[12,13]$ and Ultra-wideband (UWB) $[14,15]$, respectively corresponding to the IEEE standards 802.15.4, 802.15.1, $802.11 \mathrm{a} / \mathrm{b} / \mathrm{g} / \mathrm{h}$, and 802.15 .3 . Table 1 briefly summarizes the features of these four technologies. From the table, UWB and Wi-Fi clearly provide higher data transfer rates, but ZigBee and Bluetooth are less expensive to implement and consume less power. Wi-Fi has the longest range $(\sim 100 \mathrm{~m})$ and is designed for use in wireless local area networks (WLAN), while ZigBee, Bluetooth, and UWB are intended for short range ( 10 m) wireless personal area network (WPAN) applications, though ZigBee can reach $100 \mathrm{~m}$ in some applications.

Note that the data throughput rate is typically less than the maximum theoretical data rate due to communication overhead. Interference and distance have a strong negative impact on observed throughput. Based on the industrial context (e.g., floor area, radio interference from the surrounding metals, etc.) as well as the required sensor information, $\mathrm{Wi}-\mathrm{Fi}$ is ultimately selected for use in the proposed application.

In this work wireless sensing system was designed to monitor the health status of ball screw drives in an intelligent manufacturing environment. The developed system consists of two key modules: a temperature sensing module for data detection and amplification, and a wireless transceiver module for signal processing and transmission.

Figure 1 shows the application scenario of the proposed wireless sensor system. The coordinator at the sensor node first gathers temperature data sampled from the ball screw. This data is then sent to a computer either directly or through the access points (APs) for further diagnosis. The developed sensor module is compact enough to be embedded inside the key components so that the machine can be locally monitored. 


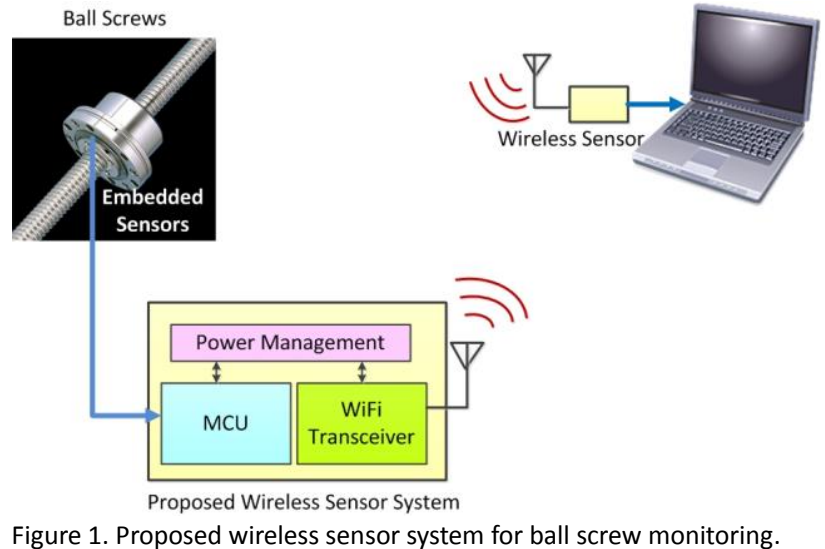

\section{Design of Wireless Sensors}

Temperature Sensing Module

To facilitate the acquisition of physical signals at specific positions, the sensing module is miniaturized ( 1 $\mathrm{cm}^{3}$ ), allowing it to be easily embedded into a ball nut close to the sliders and supporting bearings. While rod-shaped resistance- temperature-detector (RTD) type temperature sensors (e.g., PT-100) provide reliable and high resolution measurement results in industrial environments, they are too large for use in this context. Therefore, a commercially-available glass-type Pt-1000 element measuring less than $3 \mathrm{~mm} \times 3 \mathrm{~mm} \times 1 \mathrm{~mm}$, with a large temperature sensing range $\left(-200^{\sim} 350^{\circ} \mathrm{C}\right)$ is chosen for this work. Its circuitry is arranged on a FR4 printed circuit board chip, as shown in Figure 2(a). Through the Wheatstone bridge circuit configuration, the glass-type Pt-1000 element is matched with other three surface mounted device (SMD) resistors, converting the temperature change to a voltage change. The acquired signals pass through a low noise voltage amplifier to increase measurement sensitivity [16]. The whole implemented sensing module can be contained in a hole with a diameter of $1.1 \mathrm{~cm}$ and depth of $1 \mathrm{~cm}$, which is machined on the ball nut of the experimental feed drive.

Packaging is a particular challenge. The sensor circuitry was initially inserted into a metal tube to prevent interference from radiation noise from the $A C$ servo motor driver. However, static electricity induced during machine operation produces heavy signal interference. Ultimately, a thin plastic tube is used as the packaging material, sealed with epoxy, as shown in Figure 2(b). The cured epoxy can create hard coatings to prevent damage to the sensor circuitry. It also serves as good electrical insulator to reduce coupling noise.

The sensor data is sent to the amplifier module through a two-layer shielded network line, where the amplifier module is capable of processing 10 channels simultaneously, as shown in Figure 2(c).

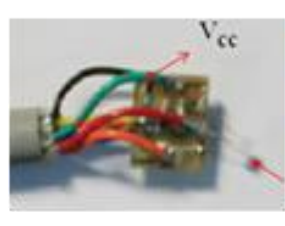

(a)

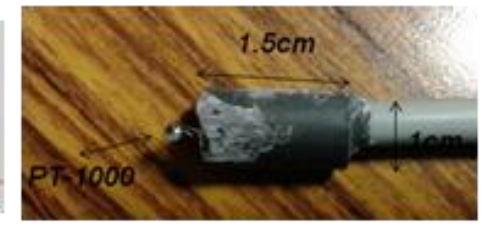

(b)

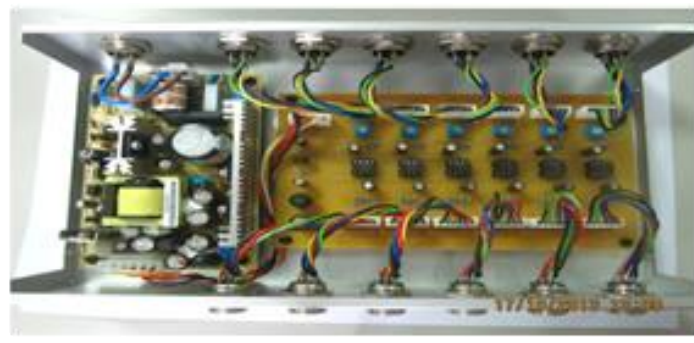

(c)

Figure 2. Temperature sensing module: (a) before packaging, (b) after packaging, (c) amplifier module.

\section{Wireless Transceiver Module}

Figure 1 also illustrates the architecture of the proposed wireless module which consists of a microcontroller (MCU), a Wi-Fi transceiver module and the power supply/management circuitry. To design this wireless transceiver module, we started with the application demo-board (APP1632, Microchip Co.) for easy control and programming. Once functionality was verified, a PCB-based Wi-Fi module was customized, providing a more compact and low-cost solution.

To simultaneously handle multiple-channel data from numerous sensor nodes, the 32-bit, up-to-80 $\mathrm{MHz}$ microprocessor (PIC32MX795F512L) from Microchip Co. is chosen here. This MCU features a 16-channel 10-bit analogy-to-digital converter with 512-kB programming Flash and 128-kB SRAM. It also allows switching between three different power modes during operation, allowing for improved power savings.

The selected wireless transceiver module (MRF24WGOMA, Microchip Co.) supports ISM Band 2.400-2.484 GHz operation, which is compatible with IEEE $802.11 \mathrm{~b} / \mathrm{g} / \mathrm{n}$ networks, with a maximum data rate of $54 \mathrm{Mbps}$. The module is designed to use the TCP/IP protocol stack, and has a built-in matching circuitry along with an integrated PCB antenna, but also provides an option for an external antenna, allowing for quick implementation. The module has a maximum transmission range greater than $100 \mathrm{~m}$.

The implementation of the integrated wireless module is shown in Figure 3 . The overall size of this PCB-based module is $6 \mathrm{~cm} \times 4.5 \mathrm{~cm}$ with UART and USB ports for data output. The module can be powered by four series-connected AA batteries ( 6 volts in total). The MAX232 and USB interfaces are powered by 5V, whereas MCU and Wi-Fi module are powered by $3.3 \mathrm{~V}$. For both interfaces to share a power source, two voltage 
regulators (MCP1825S- 5002E, Microchip Co.) are added to produce the required supply voltage for each component. Measurements show that the maximum current during the transmission mode is $320 \mathrm{~mA}$, resulting in power consumption of $1.92 \mathrm{~W}$.

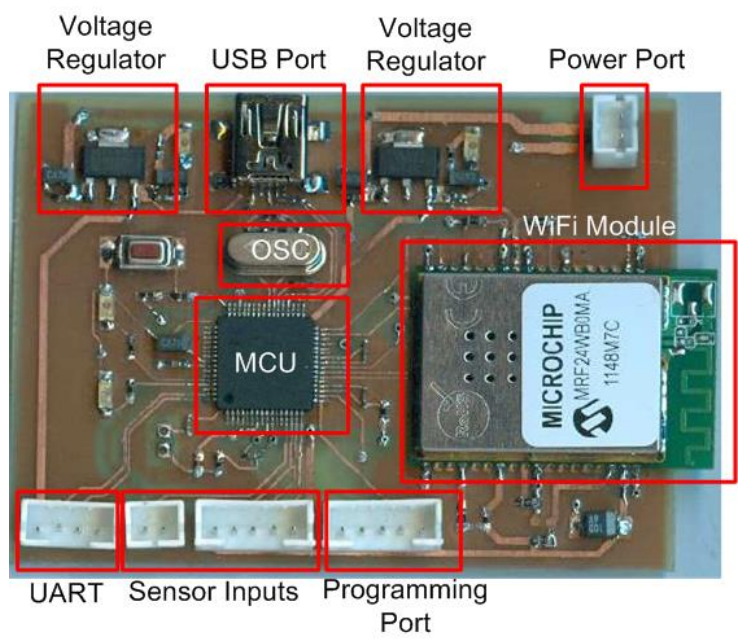

Figure 3. Integrated wireless transceiver module.

To allow the devices to connect to computers over a wireless network, the network structure should be defined. This work uses both infrastructure mode and Ad-hoc mode to increase system flexibility. In infrastructure mode, the wireless devices communicate with each other through the access points, which is a good solution for large corporate networks. Ad-hoc mode uses a direct point-to-point connection and is more suitable for small-scale networks. Either mode can be selected depending upon the size of the coverage area. With such re-configurability, this implemented wireless sensor will be able to adapt into a wide range of complex environments.

To facilitate the development and observation of sensor signals, a graphic user interface (GUI) is developed using C\# (C-sharp) programming language for data display as well as data storage, as shown in Figure 4.

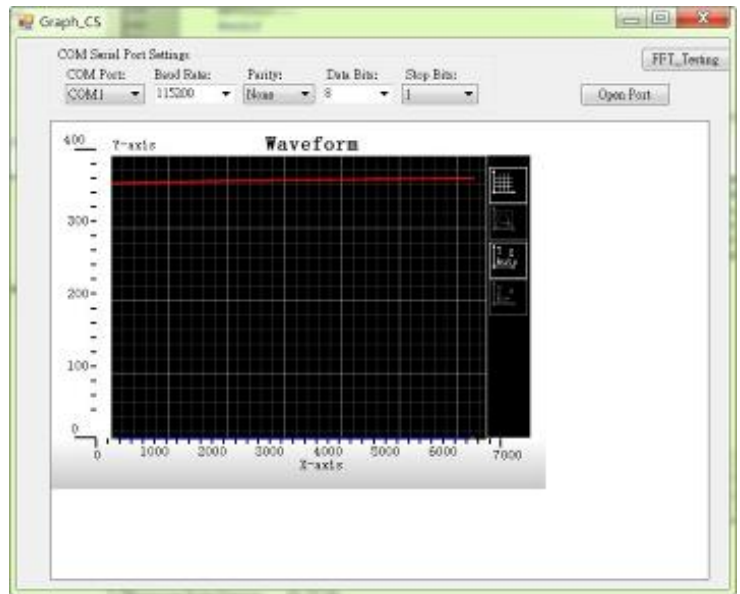

Figure 4. Developed graphic user interface based on C\# programming language.

www.ausmt.org

Copyright (c) 2014 International Journal of Automation and Smart Technology

\section{Experiments}

To evaluate the performance and functionality of this proposed wireless sensor, experiments were conducted on a ball screw stage. Figure 5 shows the experimental computer-controlled, single-axis ball screw stage (HIWIN Technologies Corp., Taichung, Taiwan). As seen in Figure 5, five $1 \mathrm{~cm} \times 1.5 \mathrm{~cm}$ cavities to hold the sensors have been pre-drilled in the following positions: 1. Left-side slider, 2. Right-side slider, 3. Ball nut, 4. Motor stage, 5. Bearing housing. These positions were chosen specifically because they all contain rich information related to the ball bearing.

In this experiment, three of the embedded sensors (left-side slider, right-side slider, and motor stage) take simultaneous readings. The measurement data are then amplified, packaged and transmitted via the wireless modules to the central computer. The computer is located in a separate room, 10-m from the machining site. Given the non-line-of-sight propagation scenario, infrastructure mode is thus applied using a mobile phone as an access point.

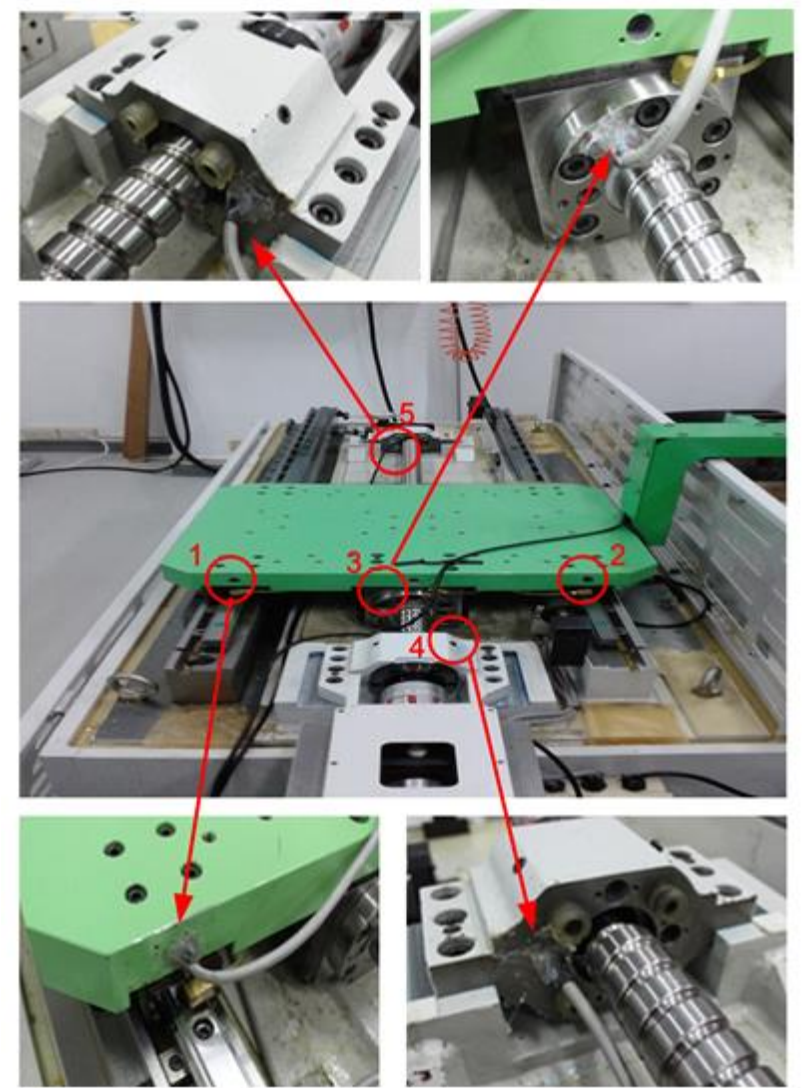

Figure 5. Wireless sensors are embedded at five different positions, including 1. Left-side slider, 2. Right-side slider, 3. Ball nut, 4. Motor stage, 5 . Bearing housing.

As seen in Figure 6(a), with the experimental stage being continuously running back and forth $(40 \mathrm{~m} / \mathrm{min}$ feed rate setting), temperatures of both sliders gradually 
increased during operation while the temperature at motor stage remained relatively constant. Based on the calibration, the voltage increase of $10 \mathrm{mV}$ represents an increase of $0.12^{\circ} \mathrm{C}$. On the other hand, Figure $6(\mathrm{~b})$ shows the collected data after the machine had been shut down. Again, a temperature drop at both sliders can be clearly observed but no such drop is found in the motor stage. Both results agree well with our expectations, since heat transfer from the ball movement has less effect on the motor stage due to the distance between them. These results can be used as a basis for comparison with the simulated thermal characteristics of the ball screw under various operation conditions, thus facilitating the development of more accurate heat transfer models. Finding the correlation between temperature distribution and related thermal deformation will facilitate future ball screw health status predictions.

Note that the glitches on the measured temperature curves are caused by the interference during the wireless transmission, which can be addressed through further signal processing. The measured data throughput of the wireless transmission is around 1.2 Mbps.

\section{Discussion and Conclusion}

A wireless sensor system is proposed, and experiment results validate the system's capability to provide network-enabled ball screw monitoring for potential use in intelligent manufacturing.

There is a trade-off between data capacity and power consumption. The power consumption in this system is still relatively high due to the use of the Wi-Fi module. Power consumption can be reduced through the appropriate application of power saving modes using the $\mathrm{MCU}$ and data transmitting algorithm, and this will be a focus of future work.

Although this paper only uses temperature sensors, we aim to simultaneously measure vibration and temperature data. An extended version of the wireless sensor is currently under development, integrating a commercially-available MEMS accelerometer (ADXL335, product of Analog Device Co.) into the reverse side of the sensor module's PCB chip. This accelerometer works according to an internal electrostatic driven scheme, where capacitance variation inside the accelerometer is associated with changes to acceleration through the output voltage. The selected sensor can simultaneously monitor accelerations along three axes, with a maximal bandwidth of $1.6 \mathrm{kHz}$ and detectable acceleration of $\pm 3 \mathrm{~g}$. Based on its lead frame chip scale package (LPCSP), the size of this accelerometer is expected to measure $4 \mathrm{~mm} \times$ $4 \mathrm{~mm} \times 1.45 \mathrm{~mm}$. This extended sensor will provide vibration information from the ball screw drives. Experimental information from the embedded sensors will be stored to a database to allow for on-line evaluation of ball screw health conditions.

This proposed wireless sensor system has numerous competitive advantages including low cost, compact size and high data rate. It can also be easily assembled with various types of sensors (e.g., humidity, voice, and light) for a wide range of applications including healthcare, home use and military.

(a)

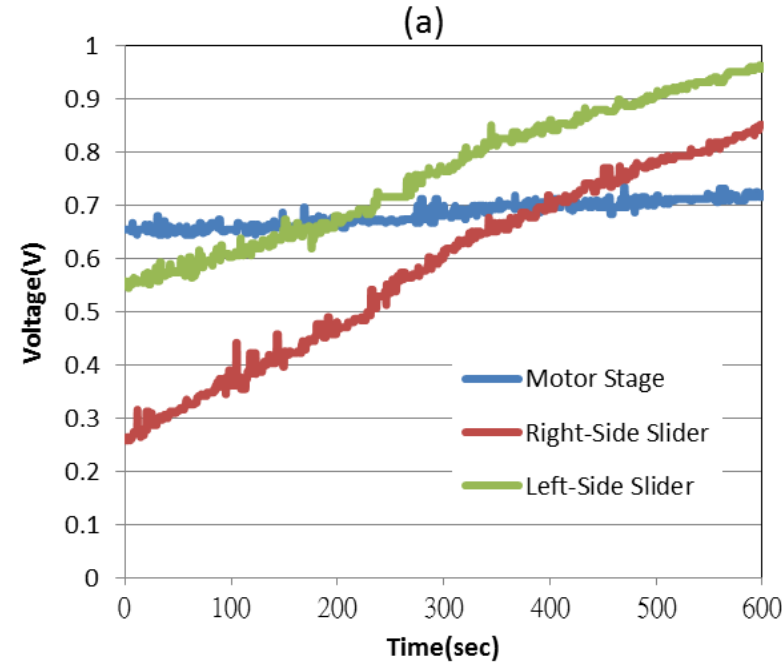

(b)

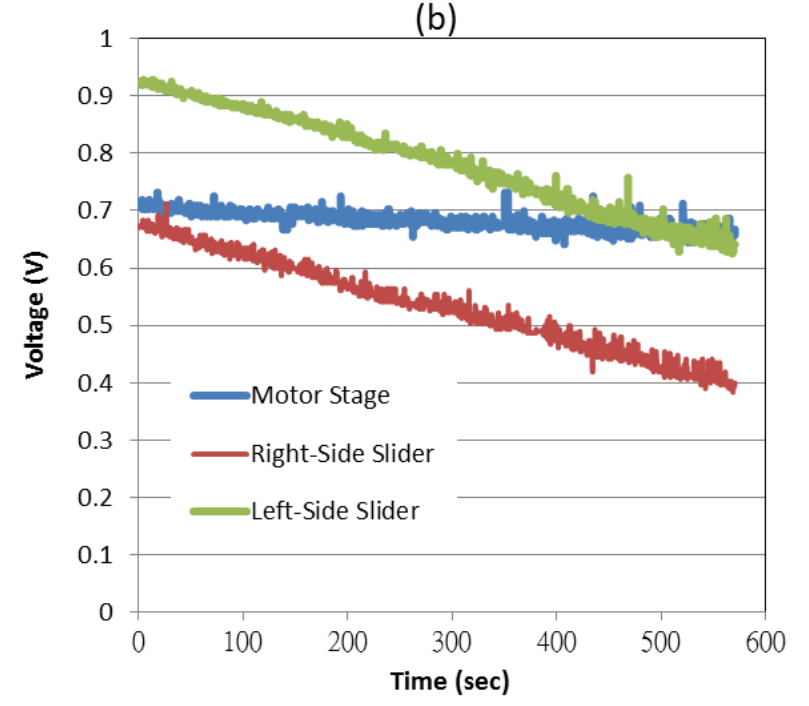

Figure 6. Measured temperature data from the sensors embedded at three different positions with the drive stage turned (a) on and (b) off.

\section{Acknowledgment}

The authors are grateful to the ROC Ministry of Economic Affairs and HIWIN Technologies Corp. for financial support provided under contract 101-EC-17-A-05-S1-189. Valuable feedback and technical assistance from HIWIN Technologies Corp. are highly appreciated. 


\section{References}

[1] Y. Altintas, A. Verl, C. Brecher, L. Uriarte, and G. Pritschow, "Machine tool feed drives," CIRP Annals - Manufacturing Technology, vol. 60, no. 2, pp. 779-796, 2011. doi: 10.1016/i.cirp.2011.05.010

[2] S. Frey, M. Walther, and A. Verl, "Periodic variation of preloading in ball screws," Production Engineering, vol. 4, no. 2-3, pp. 261-267, 2010.

doi: $10.1007 / \mathrm{s} 11740-010-0207-8$

[3] D. Kono, A. Matsubara, I. Yamaji, and T. Fujita, "High-precision machining by measurement and compensation of motion error," International Journal of Machine Tools and Manufacture, vol. 48, no. 10, pp. 1103-1110, 2008.

doi: $10.1016 /$ i.ijmachtools.2008.02.005

[4] C. W. Lee and S. W. Kim, "An ultraprecision stage for alignment of wafers in advanced microlithography," Precision Engineering, vol. 21, no. 2-3, pp. 113-122, 1997.

doi: 10.1016/S0141-6359(97)00080-9

[5] H. Nurhadi and Y. S. Tarng, "Experimental approached optimisation of a linear motion performance with grey hazy set and taguchi analysis methods (ghst) for ball-screw table type," The International Journal of Advanced Manufacturing Technology, vol. 44, no. 1-2, pp. 149-160, 2009. doi: $10.1007 / \mathrm{s} 00170-008-1816-7$

[6] M. Brown and D. G. Lowe, "Automatic panoramic image stitching using invariant features," International Journal of Computer Vision, vol. 74, no. 1, pp. 59-73, 2007.

doi: $10.1007 / \mathrm{s} 11263-006-0002-3$

[7] L. Chen and Y. Zhang, "Design and implementation of infrared wireless data transmission system," in IEEE International Conference on Information and Automation (ICIA), Harbin, China, 2010, pp. 2192-2195. doi: $10.1109 /$ ICINFA.2010.5512436

[8] M. C. Huang, J. C. Huang, J. C. You, and G. J. Jong, "The wireless sensor network for home-care system using zigbee," in 3rd International Conference on Intelligent Information Hiding and Multimedia Signal Processing, IIHMSP, Kaohsiung, Taiwan, 2007, vol. 1, pp. 643-646. doi: $\underline{10.1109 / I I H-M S P .2007 .303}$
[9] C. Cui, G. Zhao, and M. Jin, "A zigbee based embedded remote control system," in 2nd International Conference on Signal Processing Systems (ICSPS), Dalian, China, 2010, vol. 3, pp. 373-376.

doi: 10.1109/ICSPS.2010.5555814

[10] Z. Xin and G. F. Riley, "Energy-aware on-demand scatternet formation and routing for bluetooth-based wireless sensor networks," Communications Magazine, IEEE, vol. 43, no. 7 , pp. 126-133, 2005.

doi: $10.1109 /$ MCOM.2005.1470841

[11] H. Mathieu, "Wireless sensor based on bluetooth technology," INRIA, 2003.

[12] C. K. Chow and S. Y. Yuen, "A solution to illumination direction estimation of a shaded image: Genetic algorithm," Image and Vision Computing, vol. 28, no. 12, pp. 1717-1730, 2010. doi: $10.1016 /$ i.imavis.2010.06.001

[13] G. R. Mendez, M. A. M. Yunus, and S. C. Mukhopadhyay, "A wifi based smart wireless sensor network for an agricultural environment," in IEEE International Instrumentation and Measurement Technology Conference (I2MTC), Palmerston North, New Zealand, 2011, pp. 405-410.

doi: 10.1109/ICSensT.2011.6137009

[14] G. Lejiang, W. Weijiang, W. Guoshi, and C. Jian, "Research and implementation of forest fire early warning system based on uwb wireless sensor networks," in Second International Conference on Communication Systems, Networks and Applications (ICCSNA), 2010, vol. 1, pp. 176-179.

doi: 10.1109/ICCSNA.2010.5588679

[15] G. Blumrosen, M. Uziel, B. Rubinsky, and D. Porrat, "Tremor acquisition system based on uwb wireless sensor network," in International Conference on Body Sensor Networks (BSN), Singapore, 2010, pp. 187-193.

doi: $10.1109 /$ BSN.2010.28

[16] G. H. Feng and Y. L. Pan, "Investigation of ball screw preload variation based on dynamic modeling of a preload adjustable feed-drive system and spectrum analysis of ball-nuts sensed vibration signals," International Journal of Machine Tools and Manufacture, vol. 52, no. 1, pp. 85-96, 2012.

doi: 10.1016/i.ijmachtools.2011.09.008 